\title{
Comprehensive check list of Cercosporoid fungi from Iran
}

\author{
Bakhshi $\mathrm{M}^{3}$, Arzanlou $\mathrm{M}^{\mathbf{1}^{*}}$ and Babai-Ahari $\mathrm{A}^{2}$ \\ ${ }^{1}$ Assistant Professor of Plant Pathology and Mycology, Plant Protection Department, Faculty of Agriculture, University \\ of Tabriz, PO Box: 5166614766, Iran. \\ ${ }^{2}$ Professors of Plant Pathology and Mycology, Plant Protection Department, Faculty of Agriculture, University of \\ Tabriz, PO Box: 5166614766, Iran. \\ ${ }^{3}$ PhD Student of Plant Pathology, Plant Protection Department, Faculty of Agriculture, University of Tabriz, PO Box: \\ 5166614766, Iran.
}

Bakhshi M, Arzanlou M and Babai-Ahari A 2012 - Comprehensive check list of Cercosporoid fungi from Iran. Plant Pathology \& Quarantine 2(1), 44-55, doi 10.5943/ppq/2/1/7

Little is known about the biodiversity of cercosporoid fungi in Iran. We provide a comprehensive literature-based checklist for 89 cercosporoid species known to occur on different plant species in Iran. The complete annotated list covers 29 Cercospora species, 24 Ramularia, 21 Passalora and 15 Pseudocercospora.

Key words - hyphomycetes - taxonomy - mitosporic fungi

\author{
Article Information \\ Received 5 March 2012 \\ Accepted 6 March 2012 \\ Published online 10 April 2012 \\ *Corresponding author: Mahdi Arzanlou - e-mail - Arzanlou @ hotmail.com
}

\section{Introduction}

Cercosporoid fungi are generally associated with leaf spots, but also can cause necrotic lesions on flowers, fruits, bracts, seeds and pedicels of numerous hosts in a wide range of climates worldwide (Agrios 2005, Crous et al. 2000, Goodwin et al. 2001), encompassing economically important pathogens of major agricultural crops such as cereals, vegetables, ornamentals, forest trees, grasses and many others (Goodwin et al. 2001). Furthermore, a number of cercosporoid fungi are considered as potential biocontrol agents of weeds (Morris \& Crous 1994).

The genus Cercospora was established by Fresenius in 1863 . Since then many species have been assigned to this genus and there has been a significant expansion of the generic concept, including numerous Passalora-like fungi (Braun \& Melnik 1997). The first monograph of Cercospora Fresen. s. lato. was published by Chupp (1954). Later, Deighton (e.g., 1967, 1976), Braun (1995), Braun \&
Melnik (1997) and then other authors narrowed the generic concept of Cercospora s. lato. and divided it into smaller units. Crous \& Braun (2003) in their revision of cercosporoid fungi based on molecular sequence analyses and reassessment of morphological characters, recognized four true cercosporoid genera, viz. Cercospora, Pseudocercospora Speg., Passalora Fr. and Stenella Syd., and several other morphologically similar genera.

Cercospora and allied genera have traditionally been treated as anamorphs of the ascomycetous genus Mycosphaerella Johanson, (e.g., Braun \& Melnik 1997, Kim \& Shin 1998, Crous \& Braun 2003). However, a teleomorph connection for the majority of cercosporoid fungi still remains unknown, and a Mycosphaerella state has been proven for only a few species.

With the advent of molecular techniques in recent years, sequence data from different protein coding and non-coding of genomic regions have widely been applied to 
figure out phylogenetic relationships amongst wide arrays of fungal groups at different taxonomic levels of interest (Arzanlou 2007, Arzanlou 2008, Arzanlou 2010, Crous et al. 2000, Crous et al 2009). Phylogenetic analysis based on concordance of multiple sequence data have placed Cercospora and allied genera in Mycosphaerella clade (Mycosphaerellaceae, Capnodiales, Dothideomycetidae) (Crous et al. 2007, Crous et al. 2009). The genus Mycosphaerella is one of the largest genera in Ascomycetes and comprises several thousand species (Crous et al. 2001, Aptroot 2006, Crous et al. 2009).

Contrary to earlier belief in monophyly of Mycosphaerella, recent work of Crous and co-workers on phylogeny of Capnodiales revealed Mycosphaerella to be polyphyletic, and it has been split into several families; those members of Mycosphaerellaceae, Teratosphaeriaceae and Schizothyriaceae have plant pathological relevance (Crous et al. 2007, 2009). A bewildering diversity of anamorphs exists in these lineages and up to 30 anamorph genera have now been linked to Mycosphaerella (Crous et al. 2007, 2009, Arzanlou et al. 2007, 2008, Arzanlou \& Bakhshi 2012). However, recent phylogenetic analysis based on multiple sequence data sets have shown this to be incorrect. Mycosphaerellaceae, in fact, consists of numerous genera with morphologically conserved Mycosphaerella-like teleomorphs, and distinct anamorphs (Crous et al. 2007, Crous et al. 2009). Species of Mycosphaerella have a worldwide distribution from tropical and subtropical to warm and cool regions and have adapted to different ecological niches as saprobes, plant pathogens or endophytes (Farr et al. 1995, Goodwin et al. 2001, Crous et al. 2009).

Cercosporoid fungi are common and widespread on a wide range of vascular plants, especially in tropical regions. The mainland of Iran covers diverse climatic zones with a great biodiversity of vascular plants and, accordingly, a high diversity of foliicolous fungi. The exploration of this fungal group is, however, far from being complete and the cercosporoid hyphomycetes from Iran are still insufficiently known. Therefore, a comprehensive examination of cercosporoid fungi in
Iran, based on the current taxonomic classification (Crous \& Braun 2003), is urgently needed. In order to contribute to the knowledge of this group of fungi in Iran, and in the hope that what is reported here will stimulate other researchers to study the diversity of cercosporoid fungi, we have assembled a checklist of these fungi recorded to date from Iran.

\section{Materials and Methods}

\section{List of species}

The list of cercosporoid fungi was compiled using reports available in the literature. Most of the quoted works are the result of field research by Iranian mycologists, although a small number of reports have been documented by foreign investigators. The list includes cercosporoid species together with their host species from which they have been collected. The fungal nomenclature and taxonomy follows Crous \& Braun (2003). Species names used in the original publications, which deviate from those in the latter publication, are cited as synonyms. The checklist is organised alphabetically by genus and species name.

\section{Results}

A list containing 29 species of Cercospora, 21 species of Passalora, 22 species of Ramularia and 15 species of Pseudocercospora is given (Table 1). Cercospora species have been collected on 50 host plants belonging to 41 genera and 28 families. The highest numbers of cercosporoid parasites were recorded on representatives of the Fabaceae (with nine species), Malvaceae (with four species) and Euphorbiaceae (with three species). Passalora species were recordered on 29 host plants belonging to 23 genera and 14 families, including the Asteraceae (with four species) and Apiaceae (with three species), which represent the host plant families with most Passalora species. Pseudocercospora species were recorded on 18 host plants belonging to 15 genera and 12 families. The highest numbers of Pseudocercospora parasites were recorded on the Solanaceae (three species). Ramularia species were collected on 30 host plants 
Table 1 Cercosporiod fungi known from Iran.

\begin{tabular}{|c|c|c|}
\hline Species & Hosts & References \\
\hline $\begin{array}{l}\text { Cercospora } \\
\text { - acnidae } \text { Ellis \& Everh. }\end{array}$ & Amaranthus chlorostachys var. chlorostachys Willd. \& Thell. & Pirnia et al. 2010 \\
\hline - althaeina Sacc. & Althaea rosea Cav., Althaea sp. & Petrak 1956, Scharif \& Ershad 1966, Ebrahimi \& Minnasian 1973 \\
\hline - apii Fresen. & $\begin{array}{l}\text { Abutilon theophrasti Medik., Euphorbia heterophylla L., } \\
\text { Pelargonium zonale, , Solanum lycopersicum L., Vigna } \\
\text { sinensis (L.) Walp., Zanthedeschia aethiopica (L.) Spreng. }\end{array}$ & Pirnia et al. 2010 \\
\hline - beticola Sacc. & Beta maritima L., B. vulgaris L., Spinacia oleracea L. & $\begin{array}{l}\text { Esfandiari 1946a, Esfandiari 1947, Khabiri 1952, 1958, Golato 1960, } \\
\text { Eskandari 1964, Scharif \& Ershad 1966, Eskandari et al. 1969, Vinnot- } \\
\text { Bourgin et al. 1969, Altman et al. 1972, Ebrahimi \& Minnasian 1973, } \\
\text { Vaziri 1973, Alian et al. } 2008\end{array}$ \\
\hline - canescens Ellis \& Martin & Phaseolus vulgaris L., Vigna sinensis (L.) Endl & Altman et al. 1972, Ershad 1995 \\
\hline - cheiranthi Sacc. & Cheiranthus cheiri L. & Esfandiari 1948, Scharif \& Ershad 1966 \\
\hline - citrullina Cooke & Citrullus vulgaris Schrad. ex Eckl. \& Zeyh. & Scharif \& Ershad 1966 \\
\hline - datiscicola Esfand. & Datisca cannabina $\mathrm{L}$. & Esfandiari 1951 \\
\hline - elaeagni Heald \& Wolf & Elaeagnus angustifolia L. & Ershad 1995 \\
\hline - fukushiana (Mat.) Yam. & Impatiens balsamina $\mathrm{L}$. & Hedjaroude 1983 \\
\hline - gerberae Chupp \& Viegas & Gerbera jamesonii Hook. & Ershad 1995 \\
\hline - hydrangea Ellis \& Everh. & Hydrangea hortensia Siebold. & Hedjaroude 1983 \\
\hline - iridis Chupp & Iris sp. & Pirnia et al. 2010 \\
\hline -kikuchii Matsumoto \& Tomoy & Glycine $\max (\mathrm{L}$.$) Merr.$ & Zad 1979, Majidieh-Ghassemi 198 \\
\hline $\begin{array}{l}\text {-lactuca-sativae Sawada }=\text { C. longissima } \\
\text { Cooke \& Ellis }\end{array}$ & Lactuca sativa $\mathrm{L}$. & Banihashemi 1985, Pirnia et al. 2010 \\
\hline - malayensis Stev. \& Solh. & Hibiscus esculentus L. & Petrak 1956, Scharif \& Ershad 1966 \\
\hline - medicaginis Ellis \& Everh. & Medicago sp. & Scharif \& Ershad 1966 \\
\hline - mercurialis Pass. & Mercurialis annua L. & Pirnia et al. 2010 \\
\hline -musae Zimm. & Musa sapientum L. & Amani et al. 2010 \\
\hline - neriicola Ershad & Nerium oleander L. & Ershad 2002 \\
\hline -ricinella Sacc. \& Berl. & Ricinus communis L. & Petrak 1956, Khabiri 1958, Scharif \& Ershad 1966 \\
\hline -scharifii Petr. & Rosa sp. & Petrak 1956 \\
\hline - sorghi Ellis \& Everh. & Sorghum bicolor (L.) Moench, S. halepense Pers. & Mehrian \& Rajoo 2004, Mehrian 2006, Pirnia et al. 2010 \\
\hline -taurica Tranzsch. & Heliotropium sp. & Petrak 1939 \\
\hline -traversiana Sacc. & Trigonella foenum-graceum $\mathrm{L}$. & $\begin{array}{l}\text { Scharif \& Ershad } 1966 \text { as Cercospora sp., Vinnot- Bourgin et al. 1970, } \\
\text { Ershad } 1995\end{array}$ \\
\hline - viola Sacc. & $\begin{array}{l}\text { Viola odorata L., V. sintenisii Becker, V. sylvestris Lam., } \\
\text { Viola sp. }\end{array}$ & $\begin{array}{l}\text { Esfandiari 1948, Khabiri 1952, Khabiri 1958, Scharif \& Ershad 1966, } \\
\text { Vinnot-Bourgin et al. 1970, Ershad } 1995\end{array}$ \\
\hline - zebrina Pass. & Medicago sativa $\mathrm{L}$ & Ebrahimi \& Minassian 1973 \\
\hline
\end{tabular}




\begin{tabular}{|c|c|c|}
\hline Species & Hosts & References \\
\hline -zonata Wint. = C. fabae Fautr. & Vicia faba L. & $\begin{array}{l}\text { Esfandiari 1948, Scharif \& Ershad 1966, Ebrahimi \& Minnasian 1973, } \\
\text { Hedjaroude 1976, Pirnia et al. } 2010\end{array}$ \\
\hline - Cercospora spp. & $\begin{array}{l}\text { Arachis hypogaea L., Capparis spinosa L., Citrus aurantium } \\
\text { L., C. sinensis (L.) Osbeck., Citrus sp., Ficus carica L., } \\
\text { Gladiolus segetum Ker-Gawl., Glycine max (L.) Merr., } \\
\text { Hibiscus cannabinus L., Hydrangea hortensia Siebold., } \\
\text { Lactuca sativa L., Malus pumila Mill., Plantago major L., } \\
\text { Punica granatum L. }\end{array}$ & $\begin{array}{l}\text { Afzali et al. 2010, Scharif 1953, Ebrahimi 1964, Scharif \& Ershad 1966, } \\
\text { Scharif \& Akhavizadegan 1967, Daftari \& Behdad 1968, Vaziri 1973, } \\
\text { Ebrahimi \& Minassian 1973, Roohibakhsh \& Ershad } 1997\end{array}$ \\
\hline \multicolumn{3}{|c|}{ (2: } \\
\hline $\begin{array}{l}\text { - bolleana (Thum.) Braun = Cercospora } \\
\text { bolleana (Thum.) Speg. }\end{array}$ & Ficus carica $\mathrm{L}$. & $\begin{array}{l}\text { Petrak 1956, Scharif \& Ershad 1966, Vinnot- Bourgin et al. 1970, Ershad } \\
1995\end{array}$ \\
\hline $\begin{array}{l}\text { - calotropidis (Ellis \& Everh.) Braun = } \\
\text { Cercospora calotropidis Ellis \& Everh. }=C \text {. } \\
\text { patouillardi Sacc. }\end{array}$ & Calotropis procera Dryand & Petrak 1953, Petrak 1956, Vinnot- Bourgin et al. 1970, Ershad 1995 \\
\hline $\begin{array}{l}\text { - calystegiae (Speg.) Braun = Cercospora } \\
\text { calystegiae Speg. }\end{array}$ & Calystegia sp. & Hedjaroude 1983 \\
\hline $\begin{array}{l}\text { carlinae (Sacc.) Braun }=\text { Cercospora } \\
\text { carlinae Sacc. }\end{array}$ & Calina sp. & Petrak 1941 \\
\hline $\begin{array}{l}\text { - } \text { circumscissa } \text { (Sacc.) Braun = Cercospora } \\
\text { circumscissa } \text { Sacc. }\end{array}$ & $\begin{array}{l}\text { Amygdalus communis L., Cerasus avium (L.) Moench, } C \text {. } \\
\text { vulgaris Miller, Prunus domestica L. }\end{array}$ & $\begin{array}{l}\text { Petrak \& Esfandiari 1941, Esfandiari 1946a, Khabiri 1958, Vinnot- } \\
\text { Bourgin 1958, Scharif \& Ershad } 1966\end{array}$ \\
\hline - cousiniae Petr. & Cousinia nekarmanica Rech. f., Cousinia sp. & Petrak 1949, Esfandiari 1951, Jorstad 1960, Ershad 1995 \\
\hline $\begin{array}{l}\text { - dubia (Riess) Braun = Cercospora dubia } \\
\text { (Riess) Wint. }\end{array}$ & Chenopodium brumale $\mathrm{L}$. & Hedjaroude 1976 \\
\hline - fraxini (DC.) Arx & Fraxinus excelsior $\mathrm{L}$. & Kalte et al. 2008 \\
\hline $\begin{array}{l}\text {-fraxinicola (Ershad) Braun \& Crous = } \\
\text { Cercospora fraxinicola } \text { Ershad }\end{array}$ & Fraxinus rotundifolia Miller & Ershad 2000 \\
\hline $\begin{array}{l}\text { - fulva (Cooke) Braun \& Crous = Fulvia } \\
\text { fulva (Cooke) Ciferri = Cladosporium } \\
\text { fulvum Cooke. }\end{array}$ & Lycopersicum esculentum Mill. & Scharif \& Ershad 1966, Ershad 1995 \\
\hline - graminis (Fuckel) Hohn. & Glyceria fluitans $\mathrm{R} . \mathrm{Br}$. & Esfandiari 1948 \\
\hline - kirchneri (Hegyi) Petr. & Anethum graveolens L., Foeniculum vulgare Mill. & $\begin{array}{l}\text { Esfandiari \& Petrak 1950, Esfandiari 1951, Petrak 1956, Scharif \& } \\
\text { Ershad 1966, Ebrahimi \& Minassian 1973, Ershad } 1995\end{array}$ \\
\hline $\begin{array}{l}\text { - microsora (Sacc.) Braun = Cercospora } \\
\text { microsora Sacc. }\end{array}$ & Tilia begonifolis Stev., Tilia sp. & $\begin{array}{l}\text { Petrak 1941, Esfandiari 1946b, Khabiri 1958, Scharif \& Ershad 1966, } \\
\text { Ershad } 1995\end{array}$ \\
\hline $\begin{array}{l}- \text { miser (Karst.) Redhead, Vilgalys \& } \\
\text { Hopple = Coprinus miser Karst. }\end{array}$ & Salix sp. & Saber \& Esmaeili Taheri 2002 \\
\hline - phaeopappi Petr. & $\begin{array}{l}\text { Phaeopappus aucheri (DC.) Boiss; P. kotschyi (Boiss. \& } \\
\text { Heldr) Boiss }\end{array}$ & Petrak 1939, Esfandiari 1948, Golato 1960 \\
\hline
\end{tabular}


Plant Pathology \& Quarantine — Doi 10.5943/ppq/2/1/7

\begin{tabular}{|c|c|c|}
\hline Species & Hosts & References \\
\hline $\begin{array}{l}\text { - punctum (Delacr.) Petzoldt = Cercospora } \\
\text { Petroselini Sacc. }\end{array}$ & Petroselinum sativum Hoffm. & Ebrahimi \& Minassian 1973 \\
\hline $\begin{array}{l}\text { - rhamni (Fuckel) Braun }=\text { Cercospora } \\
\text { rhamni Fuckel }\end{array}$ & Rhamnus frangula $\mathrm{L}$. & Vinnot-Bourgin 1958 \\
\hline - rosae (Fuckel) Hohn. & Rosa sp. & Esfandiari 1948 \\
\hline $\begin{array}{l}\text { - scandicearum (Magnus) Braun }= \\
\text { Cercospora scandicearum Magnus }\end{array}$ & Anthriscus sylvestris Hoffm. & Vinnot-Bourgin et al. 1970 \\
\hline - scariola Syd. & Scariola orientalis (Boiss) Sojak & Petrak 1949, Esfandiari 1951 \\
\hline $\begin{array}{l}\text { - smilacis (Thum.) Braun = Cercospora } \\
\text { smilacis Thum =C. smilacina Sacc. }\end{array}$ & Smilax aspera L., S. excels L. & Petrak \& Esfandiari 1941, Esfandiari 1946b, Khabiri 1958 \\
\hline Pseudocercospora & & \\
\hline $\begin{array}{l}\text { - abelmoschi (Ellis \& Everh.) Deighton = } \\
\text { Cercospora abelmoschi Ellis \& Everh. = C. } \\
\text { hibisci Tracy \& Earle }\end{array}$ & Hibiscus cannabinus L. & $\begin{array}{l}\text { Esfandiari 1947, 1948, Scharif \& Ershad 1966, Scharif \& Akhavizadegan } \\
1967 \text { as Cercospora sp., Vinnot-Bourgin et al. } 1970\end{array}$ \\
\hline $\begin{array}{l}\text { - atromarginalis (Atk.) Deighton }= \\
\text { Cercospora atromarginalis Atk. }\end{array}$ & Solanum nigrum L. & Vinnot-Bourgin et al. 1970, Ershad 1995 \\
\hline $\begin{array}{l}\text { - cavarae (Sacc. \& D.Sacc.) Deighton = } \\
\text { Cercospora cavarae Sacc. \& D.Sacc. }\end{array}$ & Glycyrrhiza glabra L. & Hedjaroude 1983 \\
\hline $\begin{array}{l}\text { - cruenta (Sacc.) Deighton }=C \text {. cruenta } \\
\text { Sacc. }\end{array}$ & Vigna sinensis (L.) Endl. & Kaiser et al. 1968 as Cercospora sp., Vaziri 1973 \\
\hline $\begin{array}{l}\text { - fici (Heald \& Wolf) Liu \& Guo = } \\
\text { Cercospora fici Heald \& Wolf }\end{array}$ & Ficus carica $\mathrm{L}$. & Scharif \& Ershad 1966 \\
\hline - fuligena (Roldan) Deighton & Solanum nigrum L. Physalis alkekengi L. & Aghajani \& Ahmadi 2010 \\
\hline $\begin{array}{l}\text { - gomphrenae Sawada ex Goh \& Hsieh }= \\
\text { Cercospora gomphrenae }\end{array}$ & Gomphrena globosa $\mathrm{L}$. & Hedjaroude 1983 \\
\hline $\begin{array}{l}\text { - jujubae (Chowdhury) Khan \& Shamsi = } \\
\text { Cercospora jujubae Chowdhury }\end{array}$ & Ziziphus spina-christi (L.) Willd. & Ershad \& Khosravi 1996 \\
\hline $\begin{array}{l}\text {-kaki Goh \& Hsieh = Cercospora kaki Ellis } \\
\text { \& Everh. }\end{array}$ & Diospyros kaki L., D. lotus L. & $\begin{array}{l}\text { Petrak \& Esfandiari 1941, Esfandiari 1946b, Khabiri 1958, Golato 1960, } \\
\text { Scharif \& Ershad 1966, Vinnot-Bourgin et al. } 1970\end{array}$ \\
\hline $\begin{array}{l}\text { - phyllitidis (Hume) Braun \& Crous = } \\
\text { Cercospora petunia (Saito) Muller \& Chupp }\end{array}$ & Petunia var. Hort. & Ershad 1995 \\
\hline $\begin{array}{l}\text { - punicae }(\text { Henn.) Deighton }=\text { Cercospora } \\
\text { punicae Henn. }\end{array}$ & Punica granatum L. & Petrak \& Esfandiari 1941, Esfandiari 1946b, Golato 1960, Ershad 1995 \\
\hline $\begin{array}{l}\text { - rubi }(\text { Sacc.) Deighton = Cercospora rubi } \\
\text { Sacc. }\end{array}$ & Rubus sp. & Hedjaroude 1976 \\
\hline $\begin{array}{l}\text { salvadorae (Maire) Deighton }= \\
\text { Cercospora salvadorae Maire }\end{array}$ & Salvadora persica $\mathrm{L}$. & Ershad 1990 \\
\hline
\end{tabular}


Plant Pathology \& Quarantine — Doi 10.5943/ppq/2/1/7

\begin{tabular}{|c|c|c|}
\hline Species & Hosts & References \\
\hline $\begin{array}{l}\text { sphaerella-eugeniae (Sacc.) Crous, } \\
\text { Alfenas \& Barreto = Cercospora eugeniae } \\
\text { (Rangel) Chupp-Tis }\end{array}$ & Eugenia jambos L. & Ershad 1990 \\
\hline $\begin{array}{l}\text { - vitis (Lev.) Speg. = Cercospora vitis (Lev.) } \\
\text { Sacc. }\end{array}$ & Vitis sylvestris Gmel, V. vinifera L. & $\begin{array}{l}\text { Esfandiari 1947, Vinnot-Bourgin et al. 1970, Hedjaroude 1976, Ershad } \\
1990\end{array}$ \\
\hline \multicolumn{3}{|l|}{ Ramularia } \\
\hline $\begin{array}{l}\text {-alpine (Massal.) Nannf.= Ovularia alpine } \\
\text { Massal. }\end{array}$ & Alchemilla sp. & Petrak 1949, Esfandiari 1951 \\
\hline -anchusae Massal. & Anchusa italica Retz., A. ovate Lehm. & $\begin{array}{l}\text { Petrak 1953, Khabiri 1958, Vinnot-Bourgin et al. 1970, Moaven et al. } \\
2003\end{array}$ \\
\hline - beccabungae Fautr. & Veronica beccabunga L. & Petrak \& Esfandiari 1941 \\
\hline $\begin{array}{l}\text { bornmulleriana (Magnus) Braun }= \\
\text { Ovularia bornmulleriana Magnus }\end{array}$ & Onobrychis sintenisii Bornm. & Petrak 1939 \\
\hline - cynarae Sacc. $=$ R. carthami Zaprom. & Carthamus oxyacantha M. Bieb., C. tinctorius L. & $\begin{array}{l}\text { Minassian 1971, Vinnot-Bourgin et al. } 1969 \text { as Cercospora carthami } \\
\text { Sundar \& Ramakr., Altman et al. 1972, Ebrahimi \& Minassian } 1973 \text { as } \\
\text { Ramularia sp. }\end{array}$ \\
\hline - geranii Fuckel var. geranii & Geranium pyrenaicum $\mathrm{L}$. & Hedjaroude \& Abbasi 2000 \\
\hline - heraclei (Oud.) Sacc. & Heracleum persicum Desf. ex Fischer, Heracleum sp. & Esfandiari 1948, Ershad 1995 \\
\hline - iranica Petr. & Acantholimon sp. & Petrak 1949, Esfandiari 1951 \\
\hline $\begin{array}{l}\text { - lamii Fuckel = R. leonuri Sacc. \& Penz. = } \\
R . \text { menthae Sacc. }\end{array}$ & Leonurus cardiac L., Mentha arvensis L. & $\begin{array}{l}\text { Vinnot-Bourgin et al. } 1969 \text { as } R \text {. menthicola Sacc., Hedjaroude 1983, } \\
\text { Ershad 1995, Anonymous } 2005 \text { as } R \text {. menthicola Sacc. }\end{array}$ \\
\hline - macrospora Fres. & Campanula rapunculus $\mathrm{L}$. & Petrak \& Esfandiari 1941, Esfandiari 1946b, Scharif \& Ershad 1966 \\
\hline - marrubii Massal.= R. sideritis Hollos. & Sideritis $\mathrm{sp}$. & Petrak 1939 \\
\hline - primulae Thum. & Primula aqualis L. & Aghapour et al. 2010 \\
\hline $\begin{array}{l}\text { - rhabdospora (Berk. \& Broome) Nannf. = } \\
\text { R. plantaginea Sacc. }\end{array}$ & Plantago lanceolata $\mathrm{L}$. & Esfandiari 1948 \\
\hline $\begin{array}{l}\text { rubella (Bonord) Nannf. = Ovularia } \\
\text { obliqua (Cooke) Oud. }\end{array}$ & Rumex crispus L., Rumex sp. & Esfandiari 1948, Vinnot- Bourgin et al. 1969, Ershad 1995 \\
\hline - rubicola Ershad & Rubus caesius L. & Ershad 2000 \\
\hline - rufomaculans Peck & Polygonum sp. & Khabiri 1958 \\
\hline $\begin{array}{l}\text { rumicis Kalchbr. \& Cooke }=R . \text { decipiens } \\
\text { Ellis \& Everh. }\end{array}$ & Rumex crispus L., Rumex sp. & Vinnot-Bourgin et al. 1970, Ershad 1995, Fotouhifar et al. 2003 \\
\hline - rumicis-scutati Allesch. & Rumex scutatus L. & Petrak \& Esfandiari 1941, Esfandiari 1946a, Esfandiari 1946b \\
\hline - sambucina Sacc. & Sambucus ebulus L. & Petrak \& Esfandiari 1941, Esfandiari 1946b \\
\hline - simplex Pass. & Ranynchulus oxyspermus Willd. & Moaven et al. 2003 \\
\hline - uredinicola Khodap. \& Braun & Melampsora sp. & Khodaparast \& Braun 2005 \\
\hline - urticae Ces. & Urtica dioica L., U. urens L. & Esfandiari 1948, Vinnot-Bourgin 1958 \\
\hline - valeriana (Speg.) Sacc. & Valeriana sisymbrifolia Vahl. & Petrak 1939 \\
\hline Ramularia sp. & Foeniculum vulgare Mill., Potentila sp., Rumex sp. & Khabiri 1952, Vaziri 1973, Hedjaroude 1976 \\
\hline
\end{tabular}


belonging to 22 genera and 17 families, including the Polygonaceae (with five species) and Rosaceae (with three species), which represent the host plant families with most Ramularia species.

\section{Discussion}

Despite their widespread distribution, current knowledge on the cercosporoid fungi of Iran is scant. Hopefully, the information gathered here will provide both a reference work and be an incentive for further work aimed at disclosing the diversity of cercosporoid fungi in Iran.

Since the description of the genus Cercospora, the taxonomy of this genus as well as the description of individual species within this group has proven burdensome. While Cercospora was defined at genus level by morphology, species definition was based largely on host association. A significant problem pertaining to the taxonomy of Cercospora is the degree of host-specificity of the various species. Most species are still defined based on host, and they are assumed to be host-specific or restricted at least to a family of plants (Chupp 1954). However, the tenability of many species may be called into question because some taxa, including $C$. apii, the type species of Cercospora, have been shown to be non host-specific (Crous \& Braun 2003).

Most hyphomycetous genera linked to Mycosphaerella have conventionally been dealt with as part of the cercosporoid fungi (Crous \& Braun 2003). In most cases cercosporoid fungi have been treated as asexual fungi, and teleomorphs have been confirmed for only a few species. Groenewald et al. (2006) detected the two mating type genes in approximately even proportions in C. beticola, $C$. zeae-maydis and $C$. zeina populations, and speculated that a sexual cycle may occur regularly in these species. However, the actual sexual stage was not observed. Hence, the application of the criterion of intersterility is also mainly limited in cercosporoid fungi.

Iran is one of the richest biodiversity hotspots in the world. This is due to the variety of climatic zones ranging from subtropical and tropical rain forests to wetlands. Despite this wealth, however, little information is available, regarding cercosporoid fungi and their distribution as well as host range in Iran. Most publications describe the species from herbarium material and no cultures of cercosporoid fungi are present in the country.

It is difficult to rely solely on morphology for the species identification in this group. Since the combination of morphological and phylogenetic analyses of new proposed taxa in Cercospora complex is very important in order to avoid misidentification, in our opinion, future work should be focused on phylogenetic analysis of cercosporoid fungi. Hence, we are currently busy with phenotypic and molecular characterization of cercosporoid fungi of Iran based on single spore cultures.

\section{Acknowledgements}

The authors would like to thank the Research Deputy of the University of Tabriz, Iran for financial support.

\section{References}

Afzali H, Asef MR, Eskandari M 2010 Fungal diseases of pomegranate in Khorasan-Razavi and their distribution map. 19th Iranian Plant Protection Congress, July 31- August 3, Tehran, Iran.

Aghajani MA, Ahmadi MR 2010 - Black leaf mold of black nightshade and pygmy ground cherry in Iran. 19th Iranian Plant Protection Congress, July 31August 3, Tehran, Iran.

Aghapour B, Sharifi Nezamabad P, Javan Nikkhah M 2010 - A report of Ramularia primulae in Iran. 19th Iranian Plant Protection Congress, July 31August 3, Tehran, Iran.

Agrios GN 2005 - Plant pathology, $5^{\text {th }}$ ed. Academic press, San Francisco, California.

Altman J, Eslami AK, Vaziri A 1972 Diseases of crops in the Khuzestan province of southwestern Iran. Plant Disease Reporter 56, 1067-1069.

Amani M, Amani H, Zare R 2010 Identification of fungi isolated from preharvest fruit and leaf of banana in Baluchestan. 19th Iranian Plant Protection Congress, July 31-August 3, 
Tehran, Iran.

Anonymous 2005 - New hosts and distribution areas in Iran. Iranian Journal of Plant Pathology 41, 713 (in Persian) and 271 (in English).

Aptroot A 2006 - Mycosphaerella and its anamorphs: 2. Conspectus of Mycosphaerella. CBS Biodiversity Series 5, $1-231$.

Arzanlou M, Bakhshi M 2012 - Microcyclospora rumicis, a new species on Rumex crispus from Iran. Mycotaxon 118, 181186.

Arzanlou M, Abeln ECA, Kema GHJ, Waalwijk C, Carlier J, Vries Ide, Guzman M, Crous PW 2007 - Molecular diagnostics for the Sigatoka disease complex of banana. Phytopathology 97, 1112-1118.

Arzanlou M, Groenewald JZ, Fullerton RA, Abeln ECA, Carlie J, Zapater MF, Buddenhagen IW, Viljoen A, Crous PW 2008 - Multiple gene genealogies and phenotypic characters differentiate several novel species of Mycosphaerella and related anamorphs on banana. Persoonia 20, 19-37.

Arzanlou M, Crous PW, Zwiers LH 2010 Evolutionary dynamics of mating-type loci of Mycosphaerella spp. occurring on banana. Eukaryotic Cell 9, 164-172.

Banihashemi Z 1985 - Report on some new diseases in the southern Province of Iran. Iranian Journal of Plant Pathology 21, 21-22.

Braun U 1995 - A monograph of Cercosporella, Ramularia and allied genera (Phytopathogenic Hyphomycetes). Vol. 1. IHW Verlag, Eching, Germany.

Braun U, Melnik VA 1997 - Cercosporoid fungi from Russia and adjacent countries. Trudy Botanischeskogo Instituta Imeni V. L. Komarova, St. Petersburg 20, 1-130.

Chupp C 1954 - A monograph of the fungus genus Cercospora. Ithaca, New York.

Colby AS. 1920. Sooty blotch of pomaceous fruits. Transactions of the Illinois State Academy of Science 13, 139-179.

Crous PW, Aptroot A, Kang J, Braun U, Wingfield MJ 2000 - The genus
Mycosphaerella and its anamorphs. Studies in Mycology 45, 107-121.

Crous PW, Braun U 2003 - Mycosphaerella and its anamorphs: 1. Names published in Cercospora and Passalora. Centraalbureau voor Schimmelcultures, Utrecht, Netherlands.

Crous PW, Kang J, Braun U 2001 - A phylogenetic redefinition of anamorph genera in Mycosphaerella based on ITS rDNA sequence and morphology. Mycologia 93, 1081-1101.

Crous PW, Gams W, Stalpers JA, Robert V, Stegehuis G 2004 - MycoBank: an online initiative to launch mycology into the 21st century. Studies in Mycology 50, 19-22.

Crous PW, Braun U, Groenewald JZ 2007 Mycosphaerella is polyphyletic. Studies in Mycology 58, 1-32.

Crous PW, Summerell BA, Carnegie AJ, Wingfield MJ, Hunter GC, Burgess TI, Andjic V, Barber PA, Groenewald JZ 2009 - Unraveling Mycosphaerella: do you believe in genera? Persoonia 23, 99-118.

Daftari K, Behdad E 1968 - A list of plant pests and diseases of Esfahan province. Plant Pests and Diseases Research Lab of Esfahan (in Persian with Latin names of fungi).

Deighton FC 1976 - Studies on Cercospora and allied genera. VI. Pseudocercospora Speg., Pantospora Cif. and Cercoseptoria Petr. Mycological Papers 140, 1-168.

Ebrahimi AGh 1964 - Generality about plant diseases in Khuzestan. Iranian Journal of Plant Pathology 1(10), 6-13 (in Persian).

Ebrahimi AGh, Minassian V. 1973 - An index of cultivated and wild plant diseases in Khuzestan. College of Agriculture. Jundi Shapur University, Ahvaz, Iran.

Ershad D 1990 - New records of two Cercospora species for Iran. Iranian Journal of Plant Pathology 26, 41.

Ershad D 1995 - Fungi of Iran. Ministry of Agriculture, Agricultural Research Education and Extension Organization, Tehran, Iran.

Ershad D 2000 - Two new species of 
mitosporic fungi. Rostaniha 1, 1-9 (in Persian).

Ershad D 2002 - A new Cercospora species from Iran. Rostaniha 3, 47-50 (in Persian) and 15-17 (in English).

Ershad D, Khosravi V 1996 - Two fungi new to Iranian mycoflora from Baluchestan. Iranian Journal of Plant Pathology 32, 291-292.

Esfandiari E 1946a - Contribution a 1 etude de la mycoflora de 1 Iran. Plant Protection Department. Tehran (in Persian).

Esfandiari E 1946b - Deuxieme liste des fungi ramasses en Iran. Applied Entomology and Phytopathology 2, 10-16.

Esfandiari E 1947 - Les maladies des Plants cultivees et des arbres fruitiers des regions subtropicales du nord de 1 Iran. Applied Entomology and Phytopathology 5, 1-21.

Esfandiari E 1948 - A troisieme liste des fungi ramasses en Iran. Applied Entomology and Phytopathology 8, 1-15.

Esfandiari E 1951 - Quatrieme liste de fungi de 1 Iran. Applied Entomology and Phytopathology 12-13, 1-43.

Esfandiari E, Petrak F 1950 - Pilze aus Iran. Sydowia 4, 11-38.

Eskandari F 1964 - A list of plant diseases from Northern and northwestern parts of Iran. Iranian Journal of Plant Pathology 1(5), 9-15 (in Persian).

Eskandari F, Ali-Agha N, Hedjaroude GhA, Hemmati K, Rezaian MA. 1969 - Sugar beet diseases in Iran. College of Agriculture, University of Tehran, Bulletin No.106 (in Persian with English summary).

Farr DF, Bills GF, Chamuris GP, Rossman AY 1995 - Fungi on plants and plant products in the united states. St Paul, MN, USA, APS Press.

Fotouhifar KB, Hedjaroude GhA, Moussavi SM, Okhovvat SM 2003 - Study of micromycetes flora in Karaj region, Iran (2). Rostaniha 4, 79-92 (in Persian) \& 73-77 (in English).

Golato C 1960 - Micoflora Persiana, primo elenco di batteri e miceti nocivi. Rivista di Agricoltura Subtropicale e Tropicale 54(1-3), 106-113.
Goodwin SB, Dunkle LD, Zismann VL 2001 Phylogenetic analysis of Cercospora and Mycosphaerella based on the internal transcribed spacer region of ribosomal DNA. Phytopathology 91, 648-658.

Groenewald M, Groenewald JZ, Harrington TC, Abeln ECA, Crous PW 2006 Mating type gene analysis in apparently asexual Cercospora species is suggestive of cryptic sex. Fungal Genetics and Biology 43(12), 813-825

Hedjaroude GhA 1976 - Report on some formspecies of imperfect fungi of Caspian Sea area. Iranian Journal of Plant Pathology 12, 89-94 (in Persian whit English summary).

Hedjaroude GhA 1983 - Notice sur les Ascomycetes et les Deuteromycetes inedits et nouveaux pour la mycoflore de 1 Iran. Bull. Plant Protection Organization Iran 29, 78-91.

Hedjaroude GhA, Abbasi M 2000 - Study of micromycetes flora in Karaj region, Iran (1). Rostaniha 1, 103-130 (in Persian whit English summary).

Jorstad I 1960 - Iranian plants collected by Per Wendelbo in 1959, II Uredinales and some other parasitic fungi. Arbok for Universitetet i Bergen., Matematisk Naturvitenskapelig Serie 11, 1-33.

Kalte M, Taghinasab M, Chupani S 2008 Occurrence of ash tree leaf spot in Bandar-Turkaman. Proceedings of the 18 Iranian Plant Protection Congress, vol. II, 24-27 Aug., Hamedan, Iran, 66.

Khabiri E 1952 - Contribution a la mycoflore de 1 Iran. Premier liste. Revue Mycologigue 17, 154-157.

Khabiri E 1958 - Contribution a la mycoflore de 1 Iran. Troisieme liste. Revue Mycologigue 23, 408-412.

Majidieh-Ghassemi SH 1986 - Diseases of soybean and their economic importance in Iran. 8th Iranian Plant Protection Congress, 30 Aug.-4 Sept., Esfahan, Iran.

Mehrian F 2006 - Isolation of Cercospora sorghi Ellis \& Ev. (gray leaf spot) from sorghum. 17th Iranian Plant Protection Congress, 2-5 September, Karaj, Iran. 
Mehrian F, Rahjoo V 2004 - Introduction of causal agent of sorghum leaf blight. 16th Iranian Plant Protection Congress, 28 August -1 September, Tabriz, Iran.

Minassian V 1971 - New or unreported diseases in Khuzestan. Iranian Journal of Plant Pathology 6, 140-142 (in Persian with English summary).

Moaven E, Abbasi M, Soleimani MJ 2003 - A new record of Ramularia species from Iran. Rostaniha 4, 149 (in Persian) and 108 (in English).

Petrak F 1939 - Fungi in K.H Rechinger: Ergebnisse einer botanischen Reise nach dem Iran, 1937. Annalen des Naturhistorischen Museums in Wien 50, 414-541.

Petrak F 1941 - Beitrage zur kenntnis der orientalischen pilzflora. Annalen des Naturhistorischen Museums in Wien 52, 301-396.

Petrak F 1949 - Beitrage Zur Pilzflora Irans. Sydowia 3, 268-332.

Petrak F 1953 - Beitrage Zur Kennetins der Pilzflora Irans. Sydowia 7, 501-506.

Petrak F 1956 - Iranische Pilze. Sydowia 10, 117.

Petrak F, Esfandiari E 1941 - Beitrage Zur Kennetins der Iranischen Pilzflora. Annales Mycologici 39, 204-228.

Pirnia M, Zare R, Zamanizadeh HR, Khodaparast A 2010 - Contribution to the identification of Cercospora species in Iran. Rostaniha 11(2), 183-189.

Roohibakhsh A, Ershad D 1997 - An investigation on mycoflora of citrus necrotic leaf spots in western part of Mazenderan. Iranian Journal of Plant
Pathology 33, 94-110 (in Persian) \& 34 -37 (in English).

Saber M, Esmaeili Taheri A 2002 - A report on basidiomycete fungi of kashan. 15th Iranian Plant Protection Congress, 7-11 September, Kermanshah, Iran.

Scharif G 1953 - Etude morphologique et biologique de quelques champignons folicole des agrumes en Iran. These presentee a la Faculte des Science de 1 Universite de Paris pour obtenir le titre de Docteur de 1 Universite.

Scharif G, Akhavizadegan J 1967 - Fusarium foot rot of kenaf (Hibiscus cannabinus L.) in Iran. Applied Entomology and Phytopathology 26, 1-17.

Scharif G, Ershad D 1966 - A list of fungi on cultivated plants, shrubs and trees of Iran. Ministry of Agriculture. Plant Pests and Diseases Research Institute, Tehran, Iran.

Vaziri A 1973 - List of plant diseases in Dezful, Safiabad. Irrigation Department, Agricultural Research Centre.

Vinnot-Bourgin G 1958 - Contribution a la connaissance des champignons parasites de 1 Iran. Annales des Epiphyties 9, 97-210.

Vinnot-Bourgin G, Scharif G, Eskandari F 1969 - Nouvelle contribution a la connaissance des micromycetes parasites en Iran. Applied Entomology and Phytopathology 28, 3-26.

Vinnot-Bourgin G, Ale-Agha N, Ershad D 1970 - Les champignons parasites de 1 Iran. Annales Phytopathology 2(4), 689-734. 